\title{
The evolution of influenza viruses
}

\author{
Huixun Shao \\ Beijing Research Center of Laboratorial Animals, Beijing, China; huixunshao@sina.com
}

Received 7 September 2012; revised 6 October 2012; accepted 18 October 2012

\begin{abstract}
Influenza virus infection, one of the most common infectious diseases is a highly contagious airborne disease that causes an acute febrile illness and results invariable degrees of systemic symptoms, ranging from mild fatigue to respiratory failure and death. In addition to humans, influenza also infects a variety of animal species. Influenza A viruses also can infect domestic animals (pigs, horses, dogs, chickens and ducks) and some wild birds. Some of these influenza strains are species specific, but new strains of influenza may spread from other animal species to humans. These deadly strains produced 3 global pandemics in the last century, the worst of which occurred in 1918. Three pandemics of influenza have swept the world since the "Spanish" flu of 1918, the "Asian" flu pandemic of 1957 , the "Hong Kong" flu pandemic of 1968, the "Swine" flu pandemic that began in April of 2009. The pandemic of 1957 probably made more people sick than the one of 1918, but the availability of antibiotics to treat the secondary infections that are the usual cause of death resulted in a much lower death rate. An influenza pandemic occurs only when the influenza virus mutates into something dangerously unfamiliar to our immune systems and yet is able to jump from human to human through a sneeze, cough or touch. Asia is the source of many outbreaks because swines, birds and humans live under the same roof, providing opportunity for viral mixing. The best way to prevent the sickness is to get yearly injections of a vaccine that prevents influenza.
\end{abstract}

Keywords: Influenza; Viral Structure; Viral Variation; Epidemic; Pandemic; Vaccine

\section{INTRODUCTION}

Influenza was responsible for the most devastating plague in human history, which is an acute viral infection that spreads easily from person to person. It circulates worldwide causing epidemics, and it can affect any age group, while most infected people do not need medical treatment. In addition to seasonal epidemics, influenza pandemics can occur when a new influenza virus subtype emerges and then spreads easily among human beings, which is a significant public health problem.

Viruses in the family orthomyxviridase cause influenza. There are three types of influenza viruses: A, B and C. The type A viruses are the most virulent human pathogens among the three influenza types and cause the most severe disease. Influenza B almost exclusively infects humans and is less common than influenza A. The only other animals known to be susceptible to influenza $B$ infection are the seal and the ferret. This type of influenza mutates slower than type $A$ and consequently is less genetically diverse, with only one influenza B serotype [1]. As a result of this lack of antigenic diversity, a degree of immunity to influenza B is usually acquired at an early age. This reduced rate of antigenic change, combined with its limited host range, ensures that pandemics of influenza B do not occur. Influenza C virus can infect humans, dogs and pigs, sometimes causing both severe illness and local epidemics. However, influenza $\mathrm{C}$ is less common than the other types and usually only cause mild disease in children [2].

Influenza A viruses includes the avian, swine, equine and canine influenza viruses, as well as the human influenza viruses. In the mammalian species to which they are adapted, these viruses cause respiratory disease with high morbidity and low mortality rates. More severe cases can occur in conjunction with other disease or debilitation. Although many human infections are limited to conjunctivitis or mild respiratory disease, some viral strains cause severe disease and death, some viruses can become adapted to a new species and cause outbreaks, epidemics or pandemics.

\section{VIRAL STRUCTURE}

The virus particle of influenza is $80-120 \mathrm{~nm}$ in diameter and usually roughly spherical, although filamentous forms can occur. These are made of a viral envelope containing two main types of glycoproteins, 
wrapped around a central core. The central core contains the viral RNA genome and other viral proteins that package and protect this RNA. The genome of influenza A virus is composed of eight single RNA strands that code for eleven proteins, including HA, NA, nucleoprotein (NP), M1, M2, NS1, NEP (nuclear export protein, NS2), PA, PB1 (polymerase basic1), PB1-F2, PB2. Hemagglutinin (HA) and neuraminidase (NA) are the two large glycoproteins on the outside of the viral particles. HA is a lectin that mediates binding of the virus to target cells and entry of the viral genome into the target cell, while NA is involved in the release of progeny virus from infected cells, by cleaving sugars that bind the mature viral particles $[3,4]$. Thus, these proteins are targets for antiviral drugs [5]. These protein spikes of HA allow virus to infect and damage cells and are what the immune system recognizes. NA spike is used to escape the host cell, destroying it in the process. The total genome size is 13,588 bases. HA gene encodes hemagglutinin. NA gene encodes neuraminidase. NP encodes nucleoprotein. M encodes two matrix proteins (the M1 and the M2). NS encodes two distinct non-structural proteins (NS1 and NEP). PA encodes an RNA polymerase. PB1 encodes an RNA polymerase and PB1-F2 protein. PB2 encodes an RNA polymerase.

Influenza type A viruses are categorized into subtypes based on the type of two proteins on the surface of the viral envelope: HA and NA. Different influenza viruses encode for different HA and NA proteins.

Influenza A viruses are negative-sense, single-stranded, segmented RNA viruses. The several subtypes are labeled according to an HA number and an NA number. There are 17 different types of HA (HA1-HA17) and 9 different types of NA (NA1-NA9) [6]. 153 different combinations of these proteins are possible. The newest HA antigen type, identified as HA17, was isolated from yellow-shouldered bats in 2012 [7].

\section{VIRAL VARIATION}

New influenza viruses are constantly evolving by mutation or by reassortment [8]. Mutation can cause small changes in the HA and NA antigens on the surface of the virus. This is called antigenic drift, which slowly creates an increasing variety of strains until one evolves that can infect people who are immune to the pre-existing strains. This new variant then replaces the older strains as it rapidly sweeps through the human population-often causing an epidemic $[9,10]$. In contrast, when influenza viruses reassort, they acquire completely new antigens-for example by reassortment between avian strains and human strains, this is called antigenic shift. The viruses in birds are in stasis. Almost no difference in amino acid sequences of the various proteins are present in viruses separated by many decades, although the nucleic acid sequences encoding these proteins do drift. This together with the fact that the viruses seldom cause disease in their avian reservoirs show that influenza in birds is ancient and the virus has adapted to its primary host. The gene segments of influenza A virus reassort readily during mixed infection, and viruses with new combinations of genes arise frequently. Newly arising reassortants can cause major epidemics of influenza, which are capable of epidemic spread in humans. Only three subtype of HA (HA1, HA2, and HA3) and two subtype of NA (NA1 and NA2) have been bound to date in epidemic strains of human flu virus. Three influenza pandemics occurred during the last century, this virus first appeared as the cause of the great influenza epidemic of 1918-1919. The virus isolated in the epidemic of 1957 had a different subtype of both HA and NA and was called H2N2. The H2N2 virus replaced the H1N1 virus as the cause of influenza epidemics. The H2N2 virus is itself replaced by H3N2 virus beginning with the epidemic of 1968. In the 1918-1919, influenza virus was an avian virus that adapted to humans through a series of point mutations. By contrast, the 1957 and 1968 pandemic influenza viruses were the products of reassortment, that is, three genes were derived from an avian influenza virus and the remaining five genes from the previously circulating human flu viruses.

\section{AVIAN INFLUENZA (AVIAN FLU)}

Avian flu, or fowl plague, is a potentially devastating disease, predominantly of chickens and turkeys, although the virus can also affect game birds (pheasants, partridge and quail), ratites (ostrich and emu), psittacine and passerine birds. In poultry, there are two forms of disease, low pathogenicity avian influenza (LPAI) virus generally cause asymptomatic infections. In contrast, high pathogenicity avian influenza (HPAI) virus caused severe disease that can kill up to $85 \%$ - $90 \%$ of a poultry flock. The major reservoirs of influenza $\mathrm{A}$ in nature are wild ducks and other waterfowl. All of these HA and NA subtypes have been detected in waterfowl. Influenza replicates in the lung and in the gut of birds and the infection is normally asymptomatic, but epidemics of fatal influenza have occurred in turkeys and chickens, and H5N1 virus has caused fatal infection in a number of different bird species. Some strains such as H9N2, H7N7 are highly virulent to poultry and may cause more extreme symptoms and significant mortality [11,12]. Water fowl (most notably wild ducks) can excrete virus in feces, infecting other ducks via contaminated water, then spread the virus around the world. Migratory water fowl is the natural reservoir of avian flu viruses, and these birds are also the most resistant to infection. In addition numerous deaths have been reported in wild birds. At present, HPAI is an avian disease, and there is no evidence sug- 
gesting efficient human-to human transmission of HPAI. In the future, H5N1 may mutate or reassort into a strain capable of efficient human-to-human transmission.

In 2003, HPAI viruses of the H5N1 subtype appeared in poultry in several nations in Southeast Asia. The outbreaks continued to spread, H5N1 viruses reached other parts of Asia, as well as parts of Europe, the Pacific, Africa and the Middle East. As of January 2009, they have been responsible for approximately 390 human infections, generally as the result of close contact with infective chicken, about two thirds of these cases were fatal. In these years, avian flu and people avian flu, caused by H5N1 type HPAI, epidemic spread in Asia and made every country suffer from severe economic damage. H5N1 viruses have also infected and killed mammals of other species, including tigers [13], leopards, house cats [14], palm civets, a dog and a stone marten. Although natural hosts are birds, the avian flu viruses are known to cross species barriers to infect other mammals, including humans. There are fears that an avian H5N1 strain could eventually become adapted to humans, resulting in a human pandemic.

H9N2 viruses, which have become endemic in poultry in parts of Asia and the Middle East, may be of particular concern. These viruses have caused disease outbreaks in poultry in many countries $[15,16]$. Recently, they were found in pigs with respiratory disease and fatal paralysis in China [17].

\section{SWINE INFLUENZA (SWINE FLU)}

Swine flu is a highly contagious acute respiratory disease of pigs, caused by one of several swine flu viruses. The virus is spread among pigs by aerosols and direct and indirect contact, and asymptomatic carrier pigs exist. Flu outbreaks in pigs are common, especially during winter months. Swine flu viruses are most commonly of the H1N1 subtype, but other subtypes are also circulating in pigs (e.g. H1N2, H3N1, H3N2) [18]. Influenza can spread from pigs to people and from people to pigs. Cases of swine flu have most commonly occurred in people with direct exposure to pigs, but some cases of human-tohuman transmission have been reported. $15 \%$ to $25 \%$ of swine farmers might have been infected with swine flu viruses, as well as about $10 \%$ of veterinarians. Pigs can also be infected with avian flu viruses and human seasonal influenza viruses as well as swine flu viruses [19]. The H3N2 swine virus was thought to have been originally introduced into pigs by humans. Sometimes pigs can be infected with more than one virus type at a time, which can allow the genes from these viruses to mix. This can result in an influenza virus containing genes from a number of sources, called a "reassortant" virus. Although swine flu viruses are normally specific and only infect pigs, they do sometimes cross the species barrier to cause disease in humans. Outbreaks and sporadic human infection with swine flu have been occasionally reported. Generally clinical symptoms are similar to seasonal influenza but reported clinical presentation ranges broadly from asymptomatic infection to severe pneumonia resulting in death.

\section{EQUINE INFLUENZA (EI)}

$\mathrm{EI}$ is an acute, highly contagious, viral disease that can cause rapidly spreading outbreaks of respiratory disease in horses, donkeys, mules and other equine species. The disease is not generally fatal to horses, however fatalities may occur in old or infirm horses, or young foals. Transmission of the disease is usually by viral particles coughed out by an infected horse that are airborne and spread long distances to other horses. It does not need direct contact between horses as the infective particles will remain suspended in the airstream for at least 50 - 100 metres under normal conditions and further, downwind in strong winds. Moist air and moderate temperatures aid survival times of the virus and therefore efficient transmission. In addition to airborne transfer, sharing feed and water bowls, and multi horse contact by personnel will all transmit the virus effectively. This disease occurs globally, and is caused by two main strains of virus: equine- 1 (H7N7) and equine-2 (H3N8) [20]. The disease has a nearly $100 \%$ infection rate in an unvaccinated horse population with no prior exposure to the virus. Viruses that cause EI were first isolated in 1956. The viruses can cross the species barrier to cause an epizootic disease in humans, and recently in dogs.

An outbreak of EI occurred in Australia in August 2007. A total of 3376 horses on 405 properties are suspected of having the virus. Mongolia has experienced several EI outbreaks in the past few decades. Outbreaks in 2007 and 2008 caused huge losses to the local livestock industry. During 2007 and 2011, cases of EI were reported by China, France, Germany, Ireland, Mongolia, Sweden, UK and USA. The viruses identified in China, France, Germany, Ireland and the UK were characterized as equine- 2 . The viruses identified in the USA were characterized as equine- 1 .

\section{CANINE INFLUENZA (DOG FLU)}

Dog flu is influenza occurring in canine animals [21, 22], caused by varieties of influenza virus $A$, such as equine flu virus H3N8, avian flu virus H5N1 [23], which in 2004 were discovered to cause disease in dogs. Because of the lack of previous exposure to this virus, dogs have no natural immunity to this virus. Therefore, the disease is rapidly transmitted between individual dogs [24]. The H3N8 dog flu jumped from horses to dogs, but it has never infected a human. The most common presen- 
tation seen with H3N8 virus resembles kennel cough [25]. In this milder form, an initial fever is followed by a persistent cough and, sometimes, a purulent nasal discharge. More severely affected dogs exhibit a high fever with an increased respiratory rate and other signs of pneumonia or bronchopneumonia.

\section{HUMAN INFLUENZA (HUMAN FLU)}

Human flu is a respiratory infection caused by the influenza virus. Strains circulate every year, making people sick. Humans can become ill when infected with viruses from animal sources, such as avian flu virus subtypes H5N1 and H9N2 and swine flu virus subtypes H1N1 and H3N2. The primary risk factor for human infection appears to be direct or indirect exposure to infected live or dead animals or contaminated environments. Three influenza pandemics occurred in the 20th century and killed tens of millions of people, with each of these pandemics being caused by the appearance of a new strain of the virus in humans. The most famous and lethal outbreak was the 1918 influenza pandemic (Spanish flu) (type A influenza, H1N1 subtype). In 1918 and 1919, 20 to 100 million people die from flu and its complications, dead people more than died in World War One. The majority of deaths were from bacterial pneumonia, a secondary infection caused by influenza, but the virus also killed people directly, causing massive hemorrhages and edema in the lung. Later influenza pandemics were not so devastating. They included the 1957 Asian Flu (type A, H2N2 strain) and the 1968 Hong Kong Flu (type A, H3N2 strain), but even these smaller outbreaks killed millions of people. In later pandemics and this may have helped reduce mortality compared to the Spanish Flu of 1918. Often, these new strains appear when an existing flu virus spreads to humans from other animal species, or when an usually infect birds or pigs. An avian strain named H5N1 raised the concern of a new influenza pandemic, after it emerged in Asia in the 1990s, but it has not evolved to a form that spreads easily between people. Human-to-human transmission of the avian strain has been extremely rarely reported, and is considered as limited, inefficient and unsustained. However, experts warn an occurrence of "mutant avian flu" which can easily spread among humans, because the avian flu is already endemic, in particular in Asian poultry, and it is evolving in domestic and wild birds, pigs and humans. Outbreak of such mutant avian flu in the human world may have devastating consequences, H7N7, which has unusual zoonotic potential [26], and H1N2, endemic in humans, pigs and birds.

In April 2009 a novel influenza virus evolved that combined genes from human, pig, and bird flu, initially dubbed "swine flu" and also known as influenza A/ H1N1,emerged in Mexico, the United States, and several other nations. This strain is a reassortment of several strains of H1N1.Influenza A viruses circulating in pigs that have infected humans are referred to as "variant" viruses. Human infections with an influenza A (H3N2) variant $(\mathrm{H} 3 \mathrm{~N} 2 \mathrm{v})$ virus that contains the $\mathrm{M}$ gene from the influenza A (H1N1) virus were first detected in 2011, a large increase in cases of H3N2v virus infection has been identified since July 2012. The majority of H3N2v cases have been in children, although some adults have been infected, and linked to recent direct or indirect exposure to pigs. Limited, non-sustained human-to human transmission of H3N2v virus was noted in 2011, including in households and a child care setting. To date, no deaths have occurred and all cases have recovered fully.

\section{VACCINATION}

The most common vaccine is the trivalent influenza vaccine that contains purified and inactivated antigens against three viral strains, which includes material from two influenza A virus subtypes and one flu B virus strain. A vaccine formulated for one year may be ineffective in the following year. Each year, researchers develop vaccines to prevent the spread of the flu virus. The World Health Organization (WHO) holds meetings in which experts discuss what kinds of flu viruses to include in the next vaccine. Influenza vaccine is often recommended for high-risk groups, such as children and the elderly, or in people who have asthma, diabetes, heart disease, or are immuno-compromised.

Influenza vaccines can be produced in several ways, the most common method is to grow the virus in fertilized hen eggs. After purification, the virus is inactivated to produce an inactivated-virus vaccine. Alternatively, the virus can be grown in eggs until it loses virulence and the avirulent virus given as a living vaccine [27]. The effectiveness of these flu vaccines is variable. The European Union has also approved a vaccine produced by using vats of animal cells. This technique is expected to be more scalable and avoid problems with eggs, such as allergic reactions and incompatibility with strains that affect avians like chickens.

DNA vaccines are also being explored for their utility in influenza vaccines, which expressing various combinations of the viral HA or NA as well as other viral genes have been shown to be protective in animal models. Production of these vaccines is relatively safe and есоnomic and potentially rapid. More clinical studies are, however, required to fully understand the safety, immunogenicity, and effectiveness of DNA vaccines in humans [28]. Due to the inherent variation of the influenza HA, researchers aim to general a vaccine suitable for use in all ages, providing protection against currently circulating seasonal influenza as well as avian subtypes that may in future gain the ability to transmit between hu- 
mans and bring about a new pandemic, a number of attempts have been made to design influenza vaccines based on more conserved viral epitopes. One of the most studied and developed of these universal vaccines are those that target the extracellular portion of the M2 protein (M2e) $[29,30]$. M2e is not the only conserved epitope between influenza viruses and a number of other proteins have been targeted. These include nucleoprotein and polymerase proteins through $\mathrm{T}$ cell mediated approaches and also more conserved domains of HA [31]. The immunogenicity of this part of the HA molecule and its protective potential when used as the immuniting antigen is yet to be determined. In 2011, there was some research success towards a universal vaccine that produces antibodies against proteins on the viral coat which mutate less rapidly, and thus a single shot could potentially provide longer-lasting protection [32].

\section{REFERENCES}

[1] Nobusawa, E. and Sato, K. (2006) Comparison of the mutation rates of human influenza A and B viruses. Journal of Virology, 80, 3675-3678. doi:10.1128/JVI.80.7.3675-3678.2006

[2] Matsuzaki, Y., Katsushima, N., Nagai, Y., Shoji, M., Itagaki, T., Sakamoto, M., Kitaoka, S., Mizuta, K. and Nishimura, H. (2006) Clinical features of influenza C virus infection in children. Journal of Infectious Diseases, 193, 1229-1235. doi:10.1086/502973

[3] Suzuki, Y. (2005) Sialobiology of influenza: Molecular mechanism of host range variation of influenza viruses. Biological and Pharmaceutical Bulletin, 28, 399-428. doi:10.1248/bpb.28.399

[4] Bao, Y., Bolotov, P., Dernovey, D., Kiryutin, B., Zaslavsky, L., Tatusova, T., Ostell, J. and Lipman, D. (2008) The influenza virus resource at the national center for biotechnology information. Journal of Virology, 82, 596-601. doi:10.1128/JVI.02005-07

[5] Wilson, J.C. and von Ilzstein, M. (2003) Recent strategies in the search for new anti-influenza therapies. Current Drug Targets, 4, 389-408. doi:10.2174/1389450033491019

[6] Fouchier, R.A., Munster, V., Wallensten, A., Bestebroer, T.M., Herfst, S., Smith, D., et al. (2005) Characterization of a novel influenza A virus hemagglutinin subtype (H16) obtained from black-headed gulls. Journal of Virology, 79, 2814-2822. doi:10.1128/JVI.79.5.2814-2822.2005

[7] Tong, S., Li, Y., Rivailler, P., Conrardy, C., Castitlo, D. A.A., Chen, L.-M., Recuenco, S., Etlison, J.A., et al. (2012) A distinct lineage of influenza A virus from bats. Proceedings of the National Academy of Sciences.

[8] Hay, A.J., Gregory, V., Douglas, R., Lin, Y.P. (2001) The evolution of human influenza viruses. Philosophical Transactions of the Royal Society B: Biological Sciences, 356, 1861-1870. http://www.ncbi.nlm.nih.gov/pubmed/11779385

[9] Wolf, Y.I., Viboud, C., Holmes, E.C., Koonin, E.V. and
Lipman, D.J. (2006) Long intervals of stasis punctuated by bursts of positive selection in the seasonal evolution of influenza A virus. Biology Direct, 1, 34. doi:10.1186/1745-6150-1-34

[10] Parrish, C.R. and Kawaoka, Y. (2005) The origins of new pandemic viruses: The acquisition of new host ranges by canine parvovirus and influenza A viruses. Annual Review of Microbiology, 59, 553-586. doi:10.1146/annurev.micro.59.030804.121059

[11] Li, K.S., Guan, Y., Wang, J., Smith, G., Xu, K., Duan, L., Rahardjo, A.P., Puthavathana, P., Buranathai, C., Nguyen, T., Estoepangestie, A., Chaisingh, A., Auewarakul, P., Long, H., Hanh, N., Webby, R., Poon, L.L., Chen, H., Shortridge, K.F., Yuen, K.Y., Webster, R.G. and Peiris, J.S. (2004) Genesis of a highly pathogenic and potentially pandemic H5N1 influenza virus in eastern Asia. Nature, 430 209-213. doi:10.1038/nature02746

[12] Elbers, A.R., Koch, G. and Bouma, A. (2005) Performance of clinical signs in poultry for the detection of outbreaks during the avian influenza A (H7N7) epidemic in the Netherlands in 2003. Avian Pathology, 34, 181-187. doi:10.1080/03079450500096497

[13] Mushtaq, M.H., Juan, H., Jiang, P., et al. (2008) Complete genome analysis of a highly pathogenic H5N1 influenza A virus isolated from a tiger in China. Archives of Virology, 153, 1568-1574. doi:10.1007/s00705-008-0145-3

[14] Kuiken, T., Rimmelzwaan, G., van Riel. O., et al. (2004) Avian H5N1 influenza in cats. Science, 306, 241. doi:10.1126/science.1102287

[15] Banet-Noach, C., Perk, S., Simanov, L., Grebenyuk, N., Rozenblut, E., Pokamunski, S., Pirak, M., Tendler, Y. and Panshin, A. (2007) H9N2 influenza viruses from Israeli poultry: A five-year outbreak. Avian Diseases, 51, 290 296. doi:10.1637/7590-040206R1.1

[16] Naeem, K., Siddiquea, N., Ayazb, M. and Jalaleec, M.A. (2007) Avian influenza in Pakistan: Outbreaks of low and high pathogenicity avian influenza in Pakistan during 2003-2006. Avian Diseases, 57, 189-193. doi:10.1637/7617-042506R.1

[17] Cong, Y.L., Pu, J., Liu, Q.F., Wang, S., Zhang, G.Z., Zhang, X.L., Fan, W.X., Brown, E.G. and Liu, J.H. (2007) Antigenic and genetic characterization of H9N2 swine influenza viruses in China. Journal of General Virology, 88, 2035-2041. doi:10.1099/vir.0.82783-0

[18] Kothalawala, H., Toussaint, M.J. and Gruys, E. (2006) An overview of swine influenza. Veterinary Questions, 28, 46-53.

[19] Young, K.C., Tien, D.N., Hiroichi, O., et al. (2005) Studies of H5N1 influenza virus infection of pigs by using viruses isolated in Vietnam and Thailand in 2004. Journal of Virology, 79, 10821-10825. doi:10.1128/JVI.79.16.10821-10825.2005

[20] Vincent, A.L., Ma, W., Lager, K.M., Janke, B.H. and Richt, J.A. (2008) Swine influenza viruses A North American perspective. Advances in Virus Research, 72, 127 154. doi:10.1016/S0065-3527(08)00403-X

[21] Amonsin, A., Songserm, T., Chutinimitkul, S., Jam-On, R., Sae-Herg, N., Pariyothorn, N., Payungporn, S., Theam- 
boonlers, A. and Poovorawan, Y. (2007) Genetic analysis of influenza A virus (H5N1) derived from domestic cat and dog in Thailand. Archives of Virology, 152, 19251933. doi:10.1007/s00705-007-1010-5

[22] Crawford, C., Dubovi, E.J., Donis, R.O., Castleman, W.L., Gibbs, E.P.J., Hill, R.C., Katz, J.M., Ferro, P. and Anderson, T.C. (2006) Canine influenza virus infection. Proceedings of the North American Veterinary Conference, 21 April 2007.

http://www.ivis.org/proceedings/navc/2006/SAE/218.asp ?LA=1

[23] Songserm, T., Amonsin, A., Jamon, R., Sae-Heng, N., Pariyothorn, N., Payungporn, S., Theamboonlers, A., Chutinimitkul,S., Thanawongnuwech, R. and Poovorawan, Y. (2006) Fatal avian influenza A H5N1 in a dog. Emerging Infectious Diseases, 12, 1744-1747.

[24] Buonavoglia, C. and Martella, V. (2007) Canine respiratory viruses. Veterinary Research, 38, 355-373. doi:10.1051/vetres:2006058

[25] Yoon, K.J., Cooper, V.L., Schwartz, K.J., Harmon, K.M., Kim, W.Z., Janke, B.H., Strohbehn, J., Butts, D. and Troutman, J. (2005) Influenza virus infection in racing greyhounds. Emerging Infectious Diseases, 11, 1974-1976.

[26] Fouchier, R.A., Schneeberger, P.M., Rozandaal, F.W., Broekman, J.M., Kemink, S.A., Munster, V., Kulken, T., Rimmdzuban, G.F., et al. (2004) Avian influenza A virus (H7N7) associated with human conjunctivitis and a fatal case of acute respiratory disease syndrome. Proceedings of the National Academy of Sciences, 101, 1356-1361. doi:10.1073/pnas.0308352100
[27] Ilyinskii, P.O., Thoidis, G. and Shneider, A.M. (2008) Development of a vaccine against pandemic influenza viruses: Current status and perspectives. International Reviews of Immunology, 27, 392-426. doi:10.1080/08830180802295765 http://www.ncbi.nlm.nih.gov/pubmed/19065349

[28] Drape, R.J., Macklin, M.D., Barr, I.J., Jones, S., Haynes, J.R., Dean, H.J. (2006) Epidermal DNA vaccine for influenza is immunogenic in humans. Vaccine, 24, 44754481. doi:10.1016/j.vaccine.2005.08.012

[29] Schotsaert, M., de Filette, M., Fiers, W. and Saelens, X. (2009) Universal M2 ectodomain-based influenza A vaccines: Preclinical and clinical developments. Expert Review of Vaccines, 8, 499-508. doi:10.1586/erv.09.6

[30] de Filette, M., Jou, W., Birkett, A., Lyons, K., Schultz, B. and Tonkyro, A., et al. (2005) Universal influenza A vaccine: Optimization of M2-based constructs. Virology, 337, 149-161. doi:10.1016/j.virol.2005.04.004

[31] Epstein, S.L., Kong, W.P., Misplon, J.A., Lo, C.Y., Tumpey, T.M., Xu, L., et al. (2005) Protection against multiple influenza A subtypes by vaccination with highly conserved nucleoprotein. Vaccine, 23, 5404-5410. doi:10.1016/j.vaccine.2005.04.047

[32] Ekiert, D.C., Friesen, R.H.E., Bhabha, G., Kwaks, T., Jongeneelen, M., Yu, W., Ophorst, C., Cox, F., et al. (2011) A highly conserved neutralizing epitope on group 2 influenza aviruses. Science, 333, 843-850. doi:10.1126/science.1204839 